\title{
Rogue waves in optical fibers in presence of third-order dispersion, self-steepening, and self-frequency shift
}

\author{
Adrian Ankiewicz, ${ }^{1, *}$ Jose M. Soto-Crespo, ${ }^{2}$ M. Amdadul Chowdhury, ${ }^{1}$ and Nail Akhmediev ${ }^{1}$ \\ ${ }^{1}$ Optical Sciences Group, Research School of Physics and Engineering, Institute of Advanced Studies, The Australian \\ National University, Canberra, Australian Capital Territory 0200, Australia \\ ${ }^{2}$ Instituto de Óptica, Consejo Superior de Investigaciones Cientificas (CSIC), Serrano 121, Madrid 28006, Spain \\ ${ }^{*}$ Corresponding author: ana124@physics.anu.edu.au
}

Received September 12, 2012; revised October 19, 2012; accepted October 23, 2012; posted October 31, 2012 (Doc. ID 175833); published December 6, 2012

Rogue waves in optical fibers can be mathematically described by the nonlinear Schrödinger equation and its extensions that take into account third-order dispersion, self-steepening, and self-frequency shift. These equations are integrable in special cases such as the Sasa-Satsuma or the Hirota equations. However, approximate polynomial solutions can also be obtained in cases beyond these integrable ones. We present these solutions and confirm their validity using numerical simulations. @ 2012 Optical Society of America OCIS codes: $\quad 060.5530,190.3100,190.4370,190.5530$.

\section{INTRODUCTION}

Recent advances in supercontinuum generation in optical fibers [1-9] have led to the discovery of "optical rogue waves" $[10]$. A strict definition of the latter is still a subject of intense discussion [11]. Various mechanisms of optical rogue wave formation have been considered [12-16]. In contrast to single solitons that are localized only in one dimension, in the frame moving with the group velocity, rogue waves are doubly localized waves on a nonzero background. Figuratively speaking, they are waves that "appear from nowhere and disappear without a trace" [17]. The mathematical description is mainly based on the nonlinear Schrödinger equation (NLSE) and its extensions [18-20]. Significant progress has been made on the experimental side with the recent observation of the Peregrine soliton in an optical fiber [21]. The latter is an idealized mathematical representation of a rogue wave based on the NLSE [22]. The question arises as to how this idealization changes if we add higher-order terms that are always present in the equations describing nonlinear wave propagation in an optical fiber [23,24].

The standard, dimensionless focusing NLSE can be written in the form [25]

$$
i \frac{\partial \psi}{\partial x}+\frac{1}{2} \frac{\partial^{2} \psi}{\partial t^{2}}+|\psi|^{2} \psi=0
$$

where $x$ is the normalized propagation distance along the fiber, $t$ is the retarded time in a reference frame moving with the group velocity [26,27], and the function $\psi(x, t)$ is the field envelope. Essential modifications that have to be taken into account in realistic cases include a self-steepening term, $s_{a}(\partial / \partial t)\left(|\psi|^{2} \psi\right)$, a term related to the self-frequency shift, $a_{f} \psi(\partial / \partial t)\left(|\psi|^{2}\right)$, and a third-order dispersion term, $\gamma_{3} \psi_{t t t}$ $[\underline{20}, 27]$. When these terms are added, the NLSE takes the form

$i \frac{\partial \psi}{\partial x}+\frac{1}{2} \frac{\partial^{2} \psi}{\partial t^{2}}+|\psi|^{2} \psi+i s\left[a_{f} \psi \frac{\partial}{\partial t}\left(|\psi|^{2}\right)+s_{a} \frac{\partial}{\partial t}\left(|\psi|^{2} \psi\right)-\gamma_{3} \psi_{t t t}\right]=0$.
The above-mentioned terms include the main general effects in the primary order corrections to the NLSE with the coefficients $s_{a}, a_{f}$, and $\gamma_{3}$ being three independent parameters (of order 1) controlling the relative contribution of each effect. An overall multiplicative factor, $s$, plainly represents the smallness of the three terms. Thus, effectively, only three parameters are independent. When $s=0$, the equation reduces to the NLSE, Eq. (1). Each additional term introduces asymmetry along the $t$ axis and represents a higherorder correction to the group velocity dispersion term. The effect of the three terms on solitons has been widely discussed in the literature [27-32]. When a pulse like a soliton propagates long distances along the fiber, self-frequency shift leads to pulse acceleration and self-steepening leads to asymmetric pulse reshaping, while third-order dispersion leads mainly to Cherenkov radiation. However, the influence of these terms on a short scale is not obvious. As all three terms include $t$ derivatives, the constant background is not affected by these terms. Radiation waves can be ignored as their generation occurs at a very small distance. Acceleration and reshaping effects are also small for the region where the localized solution exists. Thus, the analysis of the rogue wave should be carried out in a different manner from that normally used in soliton theory. If a rogue wave solution, even an approximate one, could be found for arbitrary values of these three parameters, the problem could be considered solved for many cases of interest. However, this is not the case.

It turns out that Eq. (2) is integrable for a few combinations of the three parameters $[28,29,33]$. In each case, the rogue wave solution can be found analytically [33-35]. However, these are exceptional cases. Extending the solutions beyond the integrable limits should significantly widen the applicability of the solutions.

The fundamental rational solution $[17, \underline{17}]$ of the NLSE Eq. (1), 

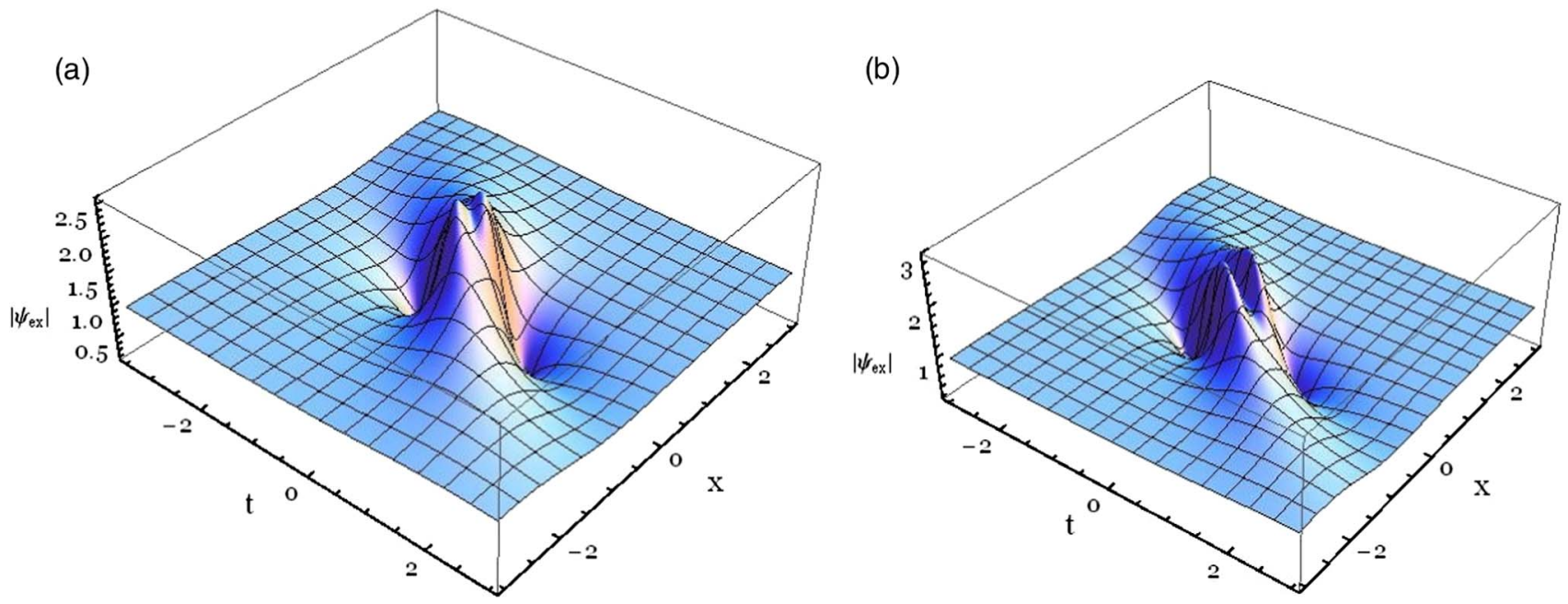

Fig. 1. (Color online) Two-dimensional profile of the exact rogue wave solution of the Sasa-Satsuma equation based on Eqs. (10) and (11) of [34]. Here $k=0$ and (a) $c=0.18, \epsilon=s=0.09$, (b) $c=0.24, \epsilon=s=0.12$.

$$
\psi=\left[4 \frac{1+2 i x}{1+4 x^{2}+4 t^{2}}-1\right] e^{i x},
$$

is commonly known as the Peregrine soliton [21]. This solution focuses the energy of the background carrier wave into a spatiotemporally localized formation due to the nonlinear properties of the fiber. In this work, we provide a few approximations relevant to the extended Eq. (2), and, using numerical simulations, we show that these approximations do describe the propagation. Of course, the accuracy depends on the values of $s$ and the three parameters chosen in the right-hand-side of Eq. (2).

Approximate results around the NLSE, to the first order in $s$, have been presented earlier in [20]. Here we consider solutions close to other integrable cases, namely the Hirota equation and the Sasa-Satsuma equation (SSE). Obtaining approximate rational solutions close to these two cases still requires certain relations between the coefficients, which we illustrate graphically. Our numerical simulations confirm that the new approximations are indeed valid.

\section{ROGUE WAVES IN THE INTEGRABLE SASA-SATSUMA CASE}

We start with the integrable Sasa-Satsuma case [28], which arises from Eq. (2) when $\gamma_{3}=1, a_{f}=3$, and $s_{a}=-6$. An exact expression for its rogue wave solution has been presented recently in [34]. The solution is quite involved, and we will not rewrite it here. The exact solution for two sets of parameters $(c=0.18, \epsilon=s=0.09)$ and $(c=0.24, \epsilon=s=0.12)$ is illustrated in Fig. 1. It has a distinct double-peak structure with the contrast between the maxima and the central amplitude depending on $\epsilon$ and $s$. Interestingly, the possibility of such structures had been also predicted using approximate solutions [20].

In order to show that this rogue wave solution may appear in a real wave field, we have numerically solved Eq. (2) using a split-step Fourier method. Figure 2 shows the evolution of the maximum field amplitude $|\psi(t)|_{\max }$ versus $x$ for the special case of the Sasa-Satsuma equation, taking as initial conditions a small bump on a constant background. The initial shape of the bump is not crucial. To be specific, in this example we used the Peregrine breather, Eq. (3), as the initial condition at $x=-6$. The values of the equation parameters used in this simulation are written within Fig. 2 . The figure clearly shows that the initial evolution up to $x=9$ describes the exact SSE rogue wave solution with its distinctive double-peak structure at the top of the solution. The maximal amplitude is also very close to the exact value of 2.5. This first stage of evolution is shown in Fig. 3(a). This figure confirms that the wave profile is indeed very similar to the shape of the exact solution presented in Fig. 1.

After $x=10$, the field evolves chaotically with several double-peak structures developed in propagation. As expected, each nearest pair of maxima are centered at different values of $t$. The amplitude profile of one of them is shown in Fig. 3(b). As the rest of the field is chaotic, the rogue wave is immersed in a sea of smaller waves.

Various initial conditions with a small perturbation of the constant background led to a similar chaotic evolution. Figure $\underline{4}$ shows a small part of the contour plot of the numerical solution of the SSE Eq. (2) with the same parameters, but with initial conditions in the form of a small random function $R(t)$ added to a constant background:

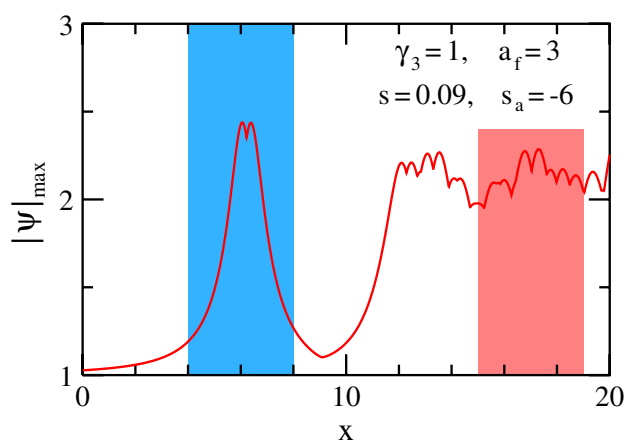

Fig. 2. (Color online) Excitation of the rogue wave of the SasaSatsuma equation (within the blue region, $4<x<8$ ) in propagation starting from the initial condition given by Eq. (3) at $x=-6$. Further evolution is chaotic with repeated double peaks, characteristic of the wave fields governed by the SSE. 

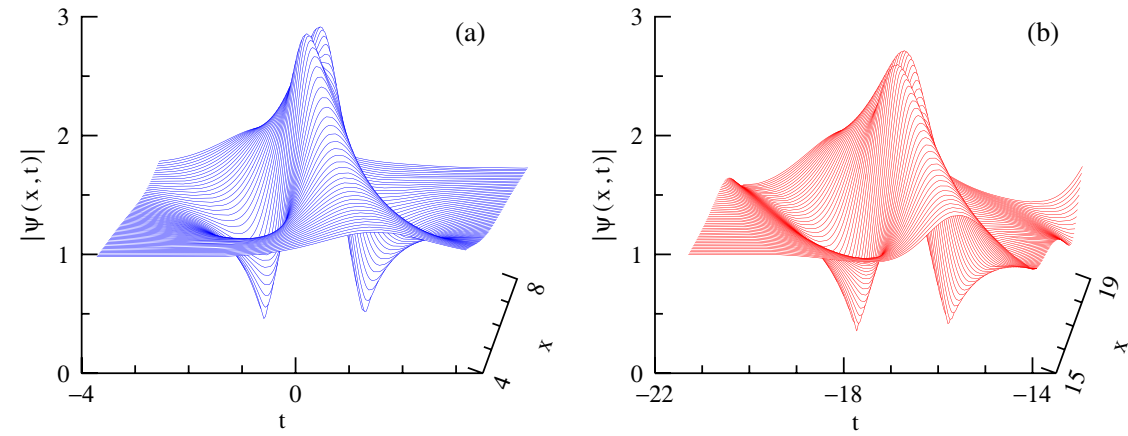

Fig. 3. (Color online) Wave profiles of the rogue waves of the Sasa-Satsuma equation within (a) the blue and (b) red regions of Fig. 2. Each one has a shape very close to that given by the exact solution and shown in Fig. 3(a).

$$
\psi(t, 0)=1+\mu R(t)
$$

where $\mu$ is a small parameter. After the initial exponential growth of perturbations, the field evolves straight into the chaotic regime without a rogue wave being excited first. However, rogue waves appear in the random field generated this way. Figure 4(a) contains two of them, which are marked by the black circles. Magnified contour plots of these two parts of the solution are shown in Figs. $\underline{4(\mathrm{~b})}$ and $\underline{4(\mathrm{c})}$. Their profiles are similar to the exact rogue wave shape but distorted by the surrounding parts of the chaotic field.

\section{APPROXIMATIONS AROUND THE SASA-SATSUMA EQUATION}

The SSE is a very particular way to extend the NLSE for a more realistic description of waves in an optical fiber. However, the integrability of this equation imposes strict values on the equation parameters. In order to partially lift this restriction, we will find approximate solutions of Eq. (2) in terms of polynomials, when the parameters are on a line that includes the SSE. However, this cannot be done easily for an arbitrary relation between the parameters of extended Eq. (2) but only on certain lines in the space of parameters. For convenience, we define a constant $k_{n}$ by

$$
k_{n}=6 \gamma_{3}+3 a_{f}+5 s_{a}
$$

Without loss of generality, we will take $\gamma_{3}=1$ and consider the plane of the two other parameters $\left(s_{a}, a_{f}\right)$. This plane is shown in Fig. 5. The integrable case of the SSE is shown on this plane by the blue diamond. The dashed line with the negative slope, which admits approximate solutions, including the SSE, is shown in blue.

It happens that we can approximate the solution around the integrable SSE case by taking $s_{a}$ as arbitrary and considering the line $a_{f}=-3-s_{a}$. Using the parameters satisfying this relation allows us to find approximate solutions. These cases are not integrable unless $s_{a}=-6$. We can write the approximate solution to the second order in $s$ in the form

$$
\begin{aligned}
\frac{\psi(x, t)}{e^{i x}}= & -1+\frac{4}{D}(1+2 i x)+\frac{8 t s}{D^{2}}\left[4 k_{n} x+i f_{a}(x, t)\right] \\
& +\frac{4 s^{2}}{D^{3}}\left[k_{b}(x, t)+2 i x f_{b}(x, t)\right],
\end{aligned}
$$
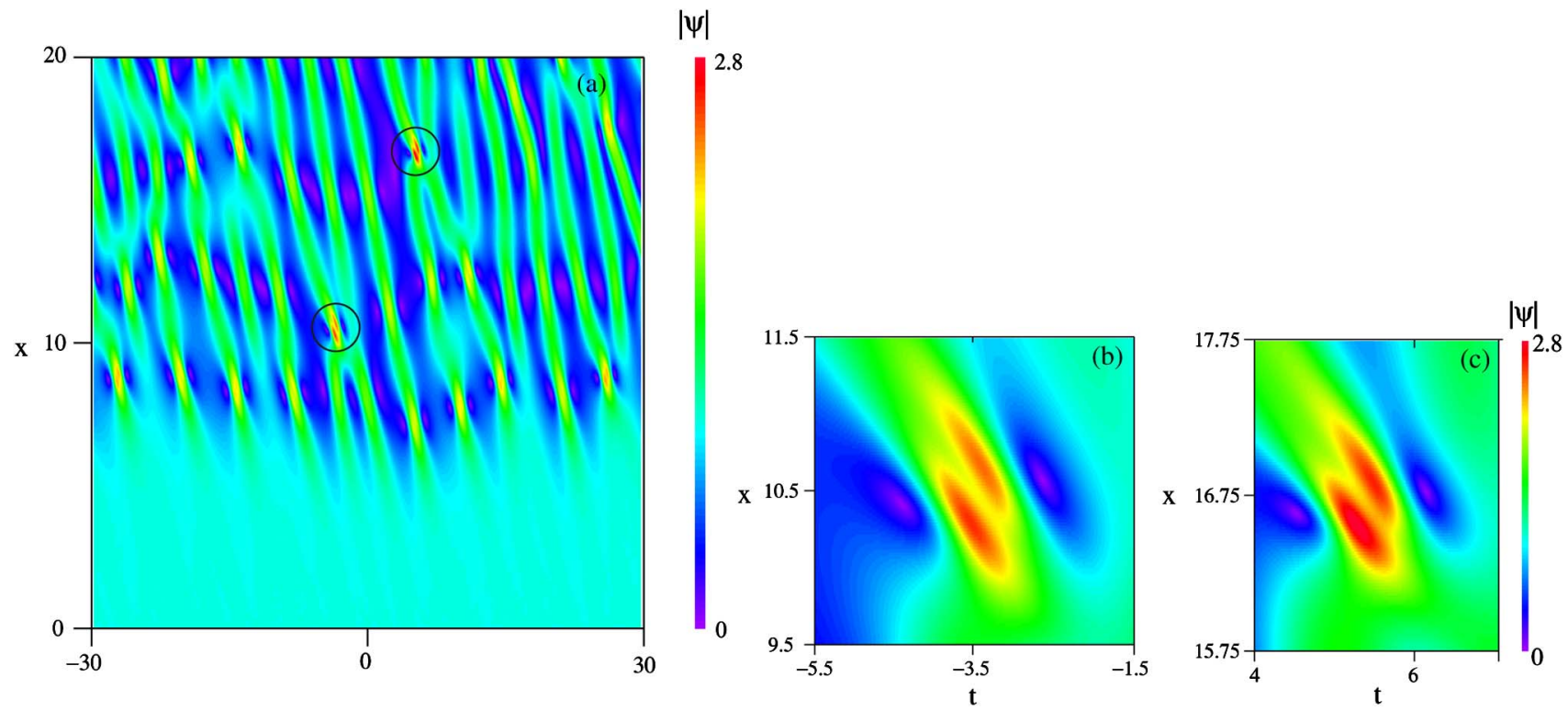

Fig. 4. (Color online) (a) Small part of the chaotic wave field generated by the SSE for an initial condition consisting of a plane wave randomly perturbed. Two rogue waves are marked by black circles. (b) and (c) represent the magnification of the encircled parts of the plot in (a). The value of the parameters is the same as in the preceding figure, namely $\gamma_{3}=1, a_{f}=3, s=0.09$, and $s_{a}=-6$. 


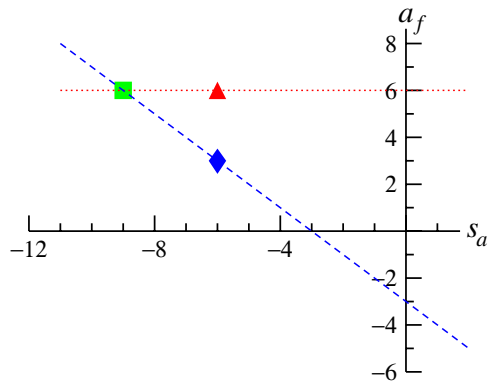

Fig. 5. (Color online) Plane of the parameters $\left(s_{a}, a_{f}\right)$ considered in this paper. With no loss of generality, we have set $\gamma_{3}=1$. The blue diamond on the sloping dashed line corresponds to the parameters of the Sasa-Satsuma equation, while the triangle directly above it indicates the parameters of the Hirota equation. The dashed blue line with negative slope gives the extension of the Sasa-Satsuma equation, while the horizontal dotted red line gives the extension of the Hirota equation. The intersection point of the lines is marked by the green square.

where $D=1+4 x^{2}+4 t^{2}$. We now find that $k_{n}=2 s_{a}-3$ and the polynomials $f_{a}(x, t), k_{b}(x, t)$ and $f_{b}(x, t)$ are

$$
\begin{aligned}
f_{a}(x, t)= & 3-s_{a}+4 t^{2}\left(s_{a}+3\right)+12 x^{2}\left(s_{a}-1\right), \\
k_{b}(x, t)= & 9\left[-1+64 t^{4}+44 x^{2}+192 x^{4}+4 t^{2}\left(3+80 x^{2}\right)\right] \\
& +3 s_{a}\left[1+48 t^{4}+24 x^{2}+80 x^{4}-16 t^{2}\left(3+8 x^{2}\right)\right] \\
& +4 s_{a}^{2}\left[4 t^{4}+t^{2}\left(28 x^{2}-3\right)-6 x^{2}\left(1+4 x^{2}\right)\right],
\end{aligned}
$$

while

$$
\begin{aligned}
f_{b}(x, t)= & 9\left[-9+32 t^{4}-20 x^{2}+64 x^{4}+4 t^{2}\left(40 x^{2}-7\right)\right] \\
& -6 s_{a}\left[1-16 x^{4}+4 t^{2}\left(5+12 x^{2}\right)\right] \\
& +s_{a}^{2}\left[3-48 x^{4}+4 t^{2}\left(4 x^{2}-9\right)\right] .
\end{aligned}
$$

The shape of this approximate rogue wave solution is shown in Fig. 6. Clearly, the shape is similar to the exact solution shown in Fig. 1. However, it changes noticeably as we vary $s_{a}$. The solution has a single peak when $s_{a}>0$, two peaks when $s_{a}<0$ (including the Sasa-Satsuma case), and up to four peaks when $s_{a} \approx-9$. The latter does not happen in the SSE case.

For the Sasa-Satsuma equation, where $s_{a}=-6$, we can add a third-order term to the right-hand-side of Eq. (6), viz.

$$
\frac{216}{D^{4}} s^{3} t\left[8 x k_{d}(x, t)-i f_{d}(x, t)\right]
$$

with

$$
\begin{aligned}
k_{d}(x, t)= & \left(4 x^{2}+1\right)\left(344 x^{2}+55\right)-32 t^{4}-4 t^{2}\left(312 x^{2}+35\right), \\
f_{d}(x, t)= & 16 t^{4}\left(16 x^{2}+23\right)+8 t^{2}\left(736 x^{4}-212 x^{2}-13\right) \\
& -8192 x^{6}-1872 x^{4}+104 x^{2}+15 .
\end{aligned}
$$

Generally, this rogue wave makes an angle $\theta$ with the $x$ axis, which means that we have an effective "velocity," $v=\tan (\theta)$. For small values of $s$ and $s_{a}$, the approximation for the velocity is

$$
v \approx\left(\frac{4}{3} s_{a}-2\right) s
$$

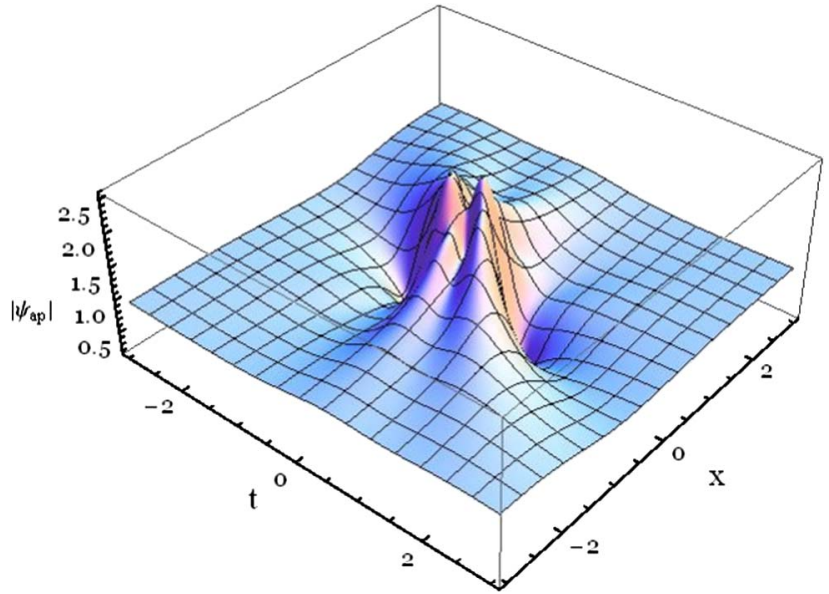

Fig. 6. (Color online) Approximation of the rogue wave near the Sasa-Satsuma equation given by Eq. (ㅁ). Here $s=0.09$.

For the Sasa-Satsuma equation, $s_{a}=-6$, and we thus have $v \approx-10 s$. This is supported by the wave directions in Figs. 1 (a) and $\underline{6}$, where we can estimate that $v \approx-0.9$.

In order to check the accuracy of our approximation, we numerically solve Eq. (2) using the approximate solution, namely Eq. ( $\underline{6})$, as the initial condition at $x=-6$. The results are shown in Fig. 7. The blue dotted curve in each panel represents the maximum of the field amplitude $|\psi(t)|_{\max }$ at each $x$, while the red dashed curve follows the maximum of the amplitude of the approximate solution Eq. (6). For small values of $s$, simulations almost coincide with the approximate solution, as we can see from Fig. 7(a). Small deviations start at $s=0.05$, as Fig. 7(b) shows. Comparison of the two rogue wave profiles for the case in Fig. 7(b) of the $x$ interval located in the yellow panel is shown in Fig. 8. The two contour plots show very similar shapes and identical velocities. The correspondence becomes poor above the value $s=0.08$, as can be seen from Fig. 7(c). We can conclude from these results that approximations on the extended line that includes the SSE are well justified for values of $s$ below 0.05 .

\section{APPROXIMATIONS AROUND THE INTEGRABLE HIROTA CASE}

The Hirota equation [29] is another integrable case when the rogue wave solution can be written in exact form $[\underline{35}, \underline{36}]$ :

$$
\frac{\psi_{e x}(x, t)}{e^{i x}}=-1+4 \frac{(1+2 i x)}{1+4(t+6 s x)^{2}+4 x^{2}} .
$$

This is a special case corresponding to the point $s_{a}=-6$. We plot Eq. (9) in Fig. 9(a) for $s=0.12$. In order to consider cases beyond the integrable one, we take arbitrary $s_{a}$ and consider the line $a_{f}=6$ (with $\gamma_{3}=1$ ). This case is depicted in Fig. $\underline{5}$ by the horizontal dotted red line. Then we can write the approximate solution in the form of polynomials:

$$
\begin{aligned}
\frac{\psi(x, t)}{e^{i x}}= & -1+\frac{4}{D}(1+2 i x)+\frac{32 t s}{D^{2}}\left[k_{n} x+i f_{e}(x, t)\right] \\
& +\frac{16 s^{2}}{D^{3}}\left[k_{c}(x, t)+2 i x f_{c}(x, t)\right]+\cdots,
\end{aligned}
$$



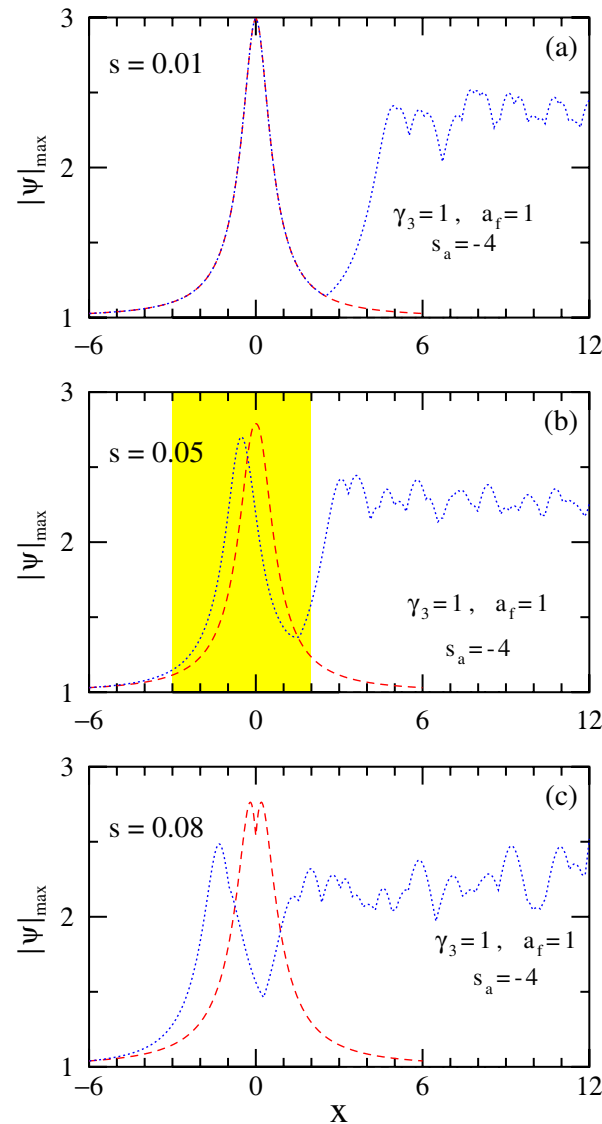

Fig. 7. (Color online) Wave propagation starting with the approximate rogue wave solution given by Eq. (6) at $x=-6$ for the parameters $s_{a}=-4$ and $a_{f}=1$. The three plots are made for (a) $s=0.01$, (b) $s=0.05$, (c) $s=0.08$. The blue dotted curve corresponds to the maximum amplitude of the field obtained in the numerical simulation, while the red dashed curve corresponds to the approximate solution Eq. (6).

where the constant $k_{n}$ in Eq. (10) is $k_{n}=24+5 s_{a}$ and the polynomials $f_{e}(x, t), k_{c}(x, t)$, and $f_{c}(x, t)$ are

$$
\begin{aligned}
f_{e}(x, t)= & \left(s_{a}+6\right)\left(2 t^{2}-1\right)+6\left(s_{a}+4\right) x^{2}, \\
k_{c}(x, t)= & s_{a}^{2}\left(32 t^{4}+12 t^{2}\left(17 x^{2}-1\right)-21 x^{2}\left(4 x^{2}+1\right)\right) \\
& +3 s_{a}\left(144 t^{4}+8 t^{2}\left(80 x^{2}-9\right)-208 x^{4}-48 x^{2}+1\right) \\
& +18\left[1+80 t^{4}+16 t^{2}\left(16 x^{2}-3\right)-8\left(6 x^{4}+x^{2}\right)\right],
\end{aligned}
$$

$|\psi|$
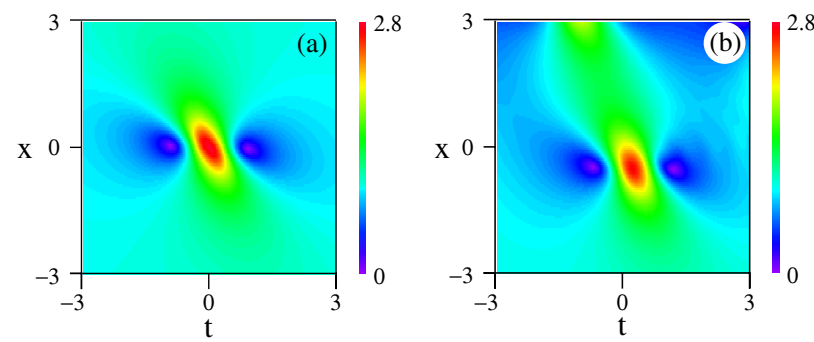

Fig. 8. (Color online) Contour plots of (a) approximate and (b) numerical profiles located within the yellow panel of Fig. 7(b)

$$
\begin{aligned}
f_{c}(x, t)= & s_{a}^{2}\left(8 t^{4}+18 t^{2}\left(2 x^{2}-3\right)-36 x^{4}+3 x^{2}+3\right) \\
& +3 s_{a}\left(32 t^{4}+4 t^{2}\left(20 x^{2}-53\right)-80 x^{4}+16 x^{2}+9\right) \\
& +18\left(16 t^{4}+8 t^{2}\left(4 x^{2}-13\right)-16 x^{4}+8 x^{2}+3\right) .
\end{aligned}
$$

For this approximate solution of the "extended Hirota equation," we find that, at small $s$, say $|s|<0.03$,

$$
v \approx\left(\frac{8}{3} s_{a}+10\right) s
$$

The special case of the Hirota equation is still included in this approximation. In this special case, $s_{a}=-6$, the velocity $v \approx-6 s$, and we can easily obtain more terms in the approximation. Then Eq. (10) can be extended to

$$
\begin{aligned}
\frac{\psi(x, t)}{e^{i x}}= & -1+\frac{4}{D}(1+2 i x)\left[1-\frac{48 x t s}{D}-\frac{144 x^{2} s^{2}}{D^{2}}\left(1-12 t^{2}\right.\right. \\
& \left.\left.+4 x^{2}\right)+\frac{13824 t x^{3} s^{3}}{D^{3}}\left(1-4 t^{2}+4 x^{2}\right)+\cdots\right] .
\end{aligned}
$$

We plot the profile defined by Eq. (12) in Fig. 9(b) for $s=0.12$.

To check the validity of the expansion in Eq. (12), we can take the exact result Eq. (9) and also expand it:

$$
\begin{aligned}
\frac{\psi_{e x}(x, t)}{e^{i x}}= & -1+\frac{4}{D}(1+2 i x)\left[1-\frac{48 x t s}{D}-\frac{144 x^{2} s^{2}}{D^{2}}\left(1-12 t^{2}+4 x^{2}\right)\right. \\
& +\frac{13824 t x^{3} s^{3}}{D^{3}}\left(1-4 t^{2}+4 x^{2}\right)+\frac{20736 x^{4} s^{4}}{D^{4}}\left(1-40 t^{2}\right. \\
& \left.\left.+80 t^{4}+8 x^{2}-160 t^{2} x^{2}+16 x^{4}\right)+\cdots\right] .
\end{aligned}
$$

Comparison with Eq. (12) shows that the approach is indeed valid. It is not surprising that Figs. 9(a) and 9(b) are very similar. Here the velocity is exactly $v=-6 s$, in agreement with the estimate given above. Consistency is seen from the fact that, at the point of intersection of the two lines shown in Fig. 5 , i.e., the point $\left(a_{f}=6, s_{a}=-9\right)$, the velocity approximations Eqs. (8) and (11) both give the same result, viz. $v=-14 s$.

In Fig. 10, we present the results of numerical simulations that start with a perturbed constant background. The perturbation is either the approximate rogue wave solution Eq. (10) at $x=-6$ (dashed blue curve) or a chaotically perturbed unit background Eq. (4) (dotted red curve). The approximate rogue wave solution is shown by the solid black curve for comparison. We can see that the approximate rogue wave solution is excited at the first stage of evolution in the case of the dashed blue curve. The contour plot of this part is presented in Fig. 10(b). Further evolution becomes chaotic, as we would expect. When the initial condition is weakly chaotic, the evolution is also chaotic after the exponential growth of the chaotic perturbation. This case is shown by the dotted red curve. These simulations confirm again that the approximate polynomial solutions describe the rogue waves reasonably well at values of $|s|<0.05$. 

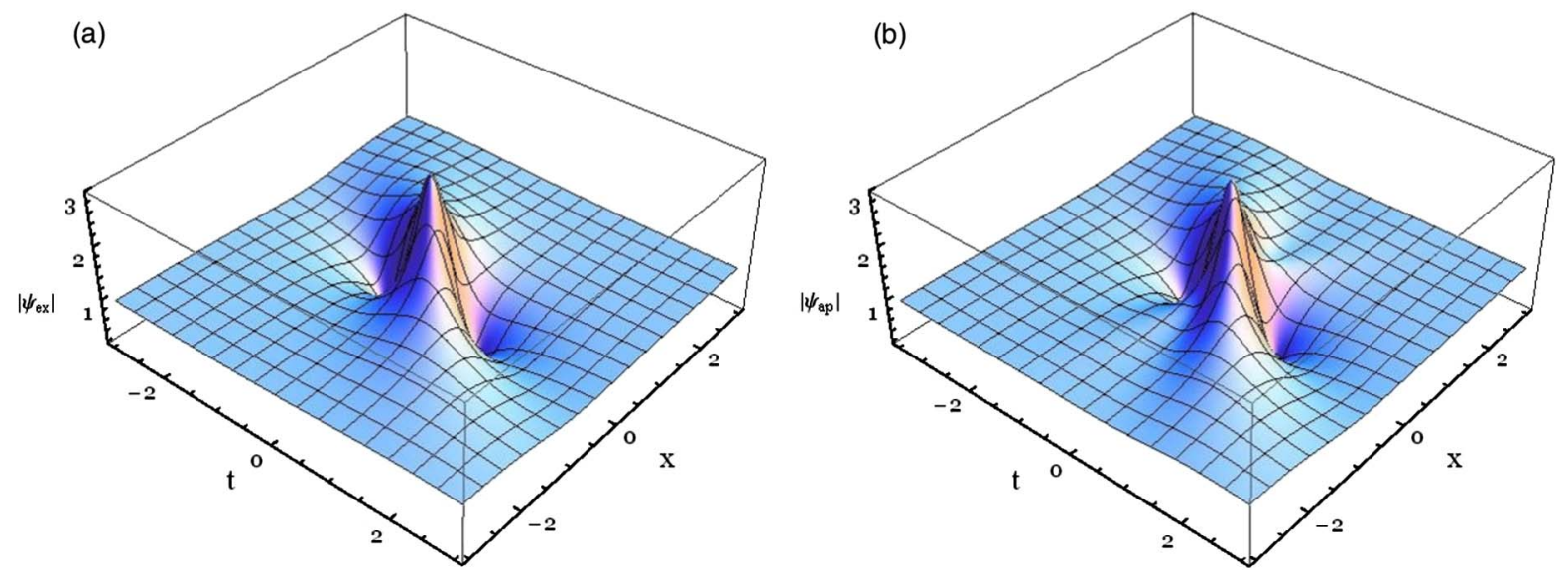

Fig. 9. (Color online) Profiles of (a) the exact rogue wave solution of the Hirota equation (9). (b) Approximation of the rogue wave of Hirota equation defined by Eq. (12). In each case, $s=0.12$.

\section{CASE WITH NO THIRD-ORDER DISPERSION}

One more case when the solution can be approximated by polynomials is when $\gamma_{3}=0$. In this case, we take $s_{a}$ as arbitrary and consider the line $a_{f}=-s_{a}$. In Fig. $\underline{5}$, this would correspond to a straight line passing through the origin. However, we should remember that here $\gamma_{3}=0$ and the above line is at a different level in the three-dimensional parameter space. Equation (2) has now effectively only one physical effect added to the NLSE:

$$
i \frac{\partial \psi}{\partial x}+\frac{1}{2} \frac{\partial^{2} \psi}{\partial t^{2}}+|\psi|^{2} \psi+i s s_{a} \psi_{t}|\psi|^{2}=0
$$

The approximate polynomial solution becomes then a function of $s s_{a}$ only:

$$
\begin{aligned}
\frac{\psi(x, t)}{e^{i x}}= & -1+\frac{4}{D}(1+2 i x)+\frac{8 t s s_{a}}{D^{2}}\left[8 x+i f_{r}(x, t)\right] \\
& +\frac{8\left(s s_{a}\right)^{2}}{D^{3}}\left[2 k_{g}(x, t)+i x f_{g}(x, t)\right] \\
& +\frac{32 t\left(s s_{a}\right)^{3}}{D^{4}}\left[2 x k_{h}(x, t)+i f_{h}(x, t)\right] \\
& +\frac{16\left(s s_{a}\right)^{4}}{D^{5}}\left[k_{j}(x, t)+2 x i f_{j}(x, t)\right]+\cdots,
\end{aligned}
$$

where the polynomials $f_{r}(x, t), k_{g}(x, t), f_{g}(x, t), k_{h}(x, t)$, $f_{h}(x, t), k_{j}(x, t)$, and $f_{j}(x, t)$ are

$$
\begin{aligned}
& f_{r}(x, t)= 4 t^{2}+12 x^{2}-1, \\
& k_{g}(x, t)= 4 t^{4}+t^{2}\left(28 x^{2}-3\right)-6 x^{2}\left(4 x^{2}+1\right), \\
& f_{g}(x, t)= 4 t^{2}\left(4 x^{2}-9\right)-48 x^{4}+3, \\
& k_{h}(x, t)=-16 t^{4}-8\left(4 t^{2}+3\right) x^{2}-24 t^{2}-144 x^{4}+3, \\
& f_{h}(x, t)=-16 t^{6}-8 t^{4}\left(8 x^{2}+1\right)+t^{2}\left(-80 x^{4}-56 x^{2}+7\right) \\
&+2 x^{2}\left(1+4 x^{2}\right)\left(19-20 x^{2}\right), \\
& k_{j}(x, t)=-192 t^{8}-32 t^{6}\left(38 x^{2}+1\right)-4 t^{4}\left(544 x^{4}+84 x^{2}-17\right) \\
&-12 t^{2} x^{2}\left(400 x^{4}-88 x^{2}-47\right) \\
&+\left(92 x^{2}-13\right) x^{2}\left(4 x^{2}+1\right)^{2}, \\
& \quad \\
& \quad+\left(20 x^{2}-31\right) x^{2}\left(4 x^{2}+1\right)^{2} . \\
& f_{j}(x, t)=304 t^{6}+8 t^{4}\left(8 x^{4}+110 x^{2}+29\right) \\
&
\end{aligned}
$$

We present the results of numerical simulations of Eq. (14) in Fig. 11 for the case $s s_{a}=0.3$. Despite the relatively large deviation from the NLSE, Eq. (15) provides a good
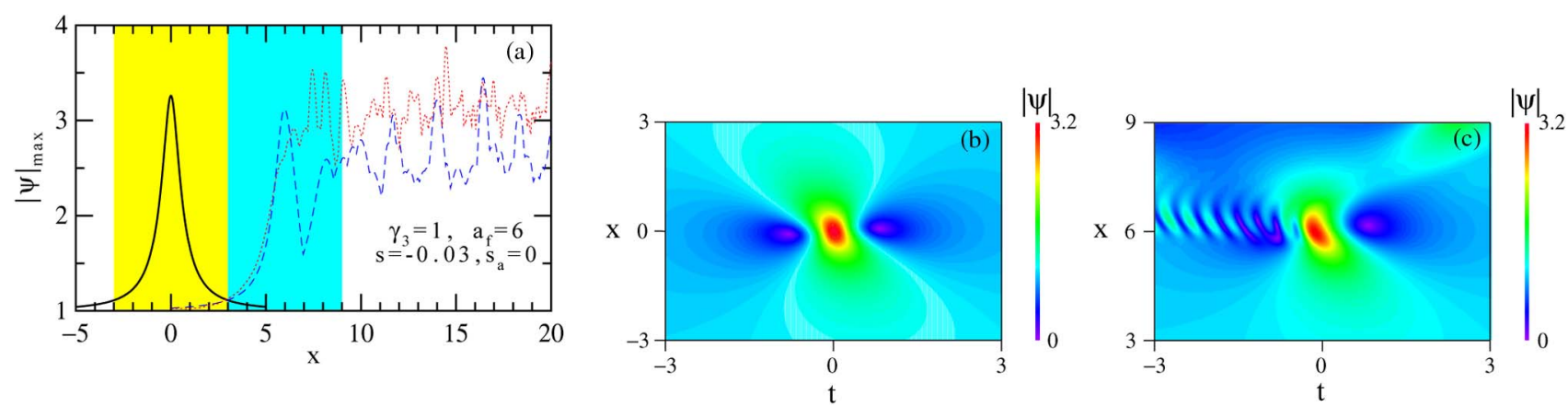

Fig. 10. (Color online) (a) Evolution of the maximum field along $x$ governed by the "extended-Hirota" equation. The solid black curve shows the maximum amplitude of the approximate solution centered at $x=0$. The dashed blue curve shows the evolution of the maximum value of the field with the initial condition Eq. (10) at $x=-6$, while the dotted red curve is for the initial condition Eq. (4). (b) Contour plot of the approximate solution in the $(x, t)$ plane [yellow part in (a), $-3<x<3$ ]. (c) Contour plot of the first peak of the blue dashed curve [light blue colored part in (a)]. 

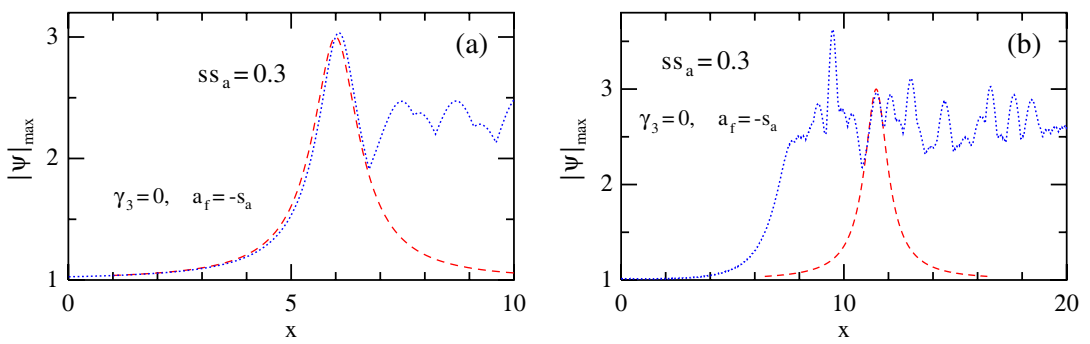

Fig. 11. (Color online) (a) Maximum of the field governed by Eq. (14), with the initial condition Eq. (15) at $x=-6$. (b) Maximum of the field governed by Eq. (14), with the initial condition as small amplitude chaotic perturbation of a constant background. Here $a_{f}=-s_{a}, \gamma_{3}=0$, and $s s_{a}=0.3$.

approximation for the solution. Figure 11(a) shows the evolution of the field maximum when the initial condition is Eq. (15) at the point $x=-6$. The red dashed curve in this figure corresponds to the approximate solution, while the blue dotted curve shows the results of simulation. As we can see, the initial stages of evolution up until $x \approx 6.7$ are very close to the approximate solution. Beyond the line $x \approx 6.7$, new waves emerge from the surrounding area, and the total field becomes chaotic. Still the first rogue wave observed in the simulations is very close to the approximate one well above $x=6.7$. The dotted blue curve deviates from the dashed red one because we are representing the maximum of the total field amplitude and, above $x \approx 6.7$, new waves emerging somewhere else from the background exceed the size of the tail of the rogue wave.

Figure $11(\mathrm{~b})$ presents the results of numerical simulations with the initial condition in the form of a constant background perturbed slightly with chaotic field Eq. (4). The evolution of the maximum in this case is chaotic with exponential growth of perturbation at the beginning. Just as in the two previous cases, some of the peaks can be identified as rogue waves very accurately described by the approximate solution of Eq. (15). To give an example, the approximate solution shown by the red dashed curve in Fig. 11(b) is adjusted close to one of these peaks.

\section{CONCLUSIONS}

In conclusion, we have presented approximate polynomial rogue wave solutions for the NLSE, taking into account third-order dispersion, self-steepening, and self-frequency shift. These solutions can be found for special relations between the coefficients of the additional terms, which include the cases when the equation is integrable (viz. the SasaSatsuma and Hirota equations). Our numerical simulations confirm the validity of the approximations when these terms are relatively small. Approximate solutions can potentially be helpful for experimentalists working on rogue waves in optical fibers [14] when short pulses are involved. Then the additional terms play an important role and must be taken into account.

\section{ACKNOWLEDGMENTS}

The authors acknowledge the support of the Australian Research Council (Discovery Project number DP110102068). The work of J. M. S. C. is supported by the MINECO under contract FIS2009-09895. A. A. and N. A. acknowledge the support from the Volkswagen Stiftung. N. A. is a recipient of the Alexander von Humboldt Award (Germany). M. A. Chowdhury is an Australian Government Endeavour Award Holder.

\section{REFERENCES}

1. J. K. Ranka, R. S. Windeler, and A. J. Stentz, "Visible continuum generation in air-silica microstructure optical fiber with anomalous dispersion at $800 \mathrm{~nm}$," Opt. Lett. 25, 25-27 (2000).

2. A. V. Husakou and J. Herrmann, "Supercontinuum generation of higher-order solitons by fission in photonic crystal fibers," Phys. Rev. Lett. 87, 203901 (2001).

3. J. M. Dudley, G. Genty, and S. Coen, "Supercontinuum generation in photonic crystal fiber," Rev. Mod. Phys. 78, 1135-1184 (2006).

4. J. Herrmann, U. Griebner, N. Zhavoronkov, A. Husakou, D. Nickel, J. C. Knight, W. J. Wadsworth, P. St. J. Russell, and G. Korn, "Experimental evidence for supercontinuum generation by fission of higher-order solitons in photonic fibers," Phys. Rev. Lett. 88, 173901 (2002).

5. J. M. Dudley and J. R. Taylor, eds., Supercontinuum Generation in Optical Fibers (Cambridge University, 2010).

6. K. M. Hilligsoe, H. N. Paulsen, J. Thogersen, S. R. Keiding, and J. J. Larsen, "Initial steps of supercontinuum generation in photonic crystal fibers,” J. Opt. Soc. Am. B 20, 1887-1893 (2003).

7. J. Bethge, A. Husakou, F. Mitschke, F. Noack, U. Griebner, G. Steinmeyer, and J. Herrmann, "Two-octave supercontinuum generation in a water-filled photonic crystal fiber," Opt. Express 18, 6230-6240 (2010).

8. A. Mussot and A. Kudlinski, "19.5 W CW-pumped supercontinuum source from 0.65 to $1.38 \mu \mathrm{m}$,” Electron. Lett. 45, 29-30 (2009).

9. J. C. Travers, "High average power supercontinuum sources," Pramana J. Phys. 75, 769-785 (2010).

10. D. R. Solli, C. Ropers, P. Koonath, and B. Jalali, "Optical rogue waves," Nature 450, 1054-1057 (2007).

11. N. Akhmediev and E. Pelinovsky, eds., Issue on "Rogue wavestowards a unifying concept?” Eur. Phys. J. Special Topics 185, 1-266 (2010).

12. D. V. Skryabin and A. V. Gorbach, "Looking at a soliton through the prism of optical supercontinuum," Rev. Mod. Phys. 82, 1287-1299 (2010).

13. G. Genty, C. M. de Sterke, O. Bang, F. Dias, N. Akhmediev, and J. M. Dudley, "Collisions and turbulence in optical rogue wave formation,” Phys. Lett. A 374, 989-996 (2010).

14. J. M. Dudley, G. Genty, F. Dias, B. Kibler, and N. Akhmediev, "Modulation instability, Akhmediev breathers and continuous wave supercontinuum generation," Opt. Express 17, 21497-21508 (2009).

15. A. Mussot, A. Kudlinski, M. Kolobov, E. Louvergneaux, M. Douay, and M. Taki, "Observation of extreme temporal events in CW-pumped supercontinuum,” Opt. Express 17, 17010-17015 (2009).

16. Ch. Mahnke and F. Mitschke, "Possibility of an Akhmediev breather decaying into solitons," Phys. Rev. A 85, 033808 (2012).

17. N. Akhmediev, A. Ankiewicz, and M. Taki, "Waves that appear from nowhere and disappear without a trace," Phys. Lett. A 373 675-678 (2009). 
18. P. Gaillard, "Families of quasi-rational solutions of the NLS equation and multi-rogue waves," J. Phys. A 44, 435204 (2011).

19. Y. Ohta and J. Yang, "General high-order rogue waves and their dynamics in the nonlinear Schrödinger equation," Proc. R. Soc. A 468, 1716-1740 (2012).

20. A. Ankiewicz, N. Devine, and N. Akhmediev, "Are rogue waves robust against perturbations?” Phys. Lett. A 373, 3997-4000 (2009).

21. B. Kibler, J. Fatome, C. Finot, G. Millot, F. Dias, G. Genty, N. Akhmediev, and J. M. Dudley, "The Peregrine soliton in nonlinear fibre optics," Nat. Phys. 6, 790-795 (2010).

22. D. H. Peregrine, "Water waves, nonlinear Schrödinger equations and their solutions," J. Aust. Math. Soc. B 25, 16-43 (1983).

23. S. B. Cavalcanti, J. C. Cressoni, Heber R. da Cruz, and A. S. Gouveia-Neto, "Modulation instability in the region of minimum group-velocity dispersion of single-mode optical fibers via an extended nonlinear Schrödinger equation," Phys. Rev. A 43, 6162-6165 (1991).

24. M. J. Potasek, "Modulation instability in an extended nonlinear Schrödinger equation," Opt. Lett. 12, 921-923 (1987).

25. N. Akhmediev and A. Ankiewicz, Solitons, Nonlinear Pulses and Beams (Chapman and Hall, 1997).

26. A. Hasegawa and F. Tappert, "Transmission of stationary nonlinear optical pulses in dispersive dielectric fibers. I. Anomalous dispersion," Appl. Phys. Lett. 23, 142-144 (1973).

27. G. P. Agrawal, Nonlinear Fiber Optics, 4th ed., Optics and Photonics series (Academic, 2006), Section 5.5.3.
28. N. Sasa and J. Satsuma, "New type of soliton solutions for a higher-order nonlinear Schrödinger equation," J. Phys. Soc. Jpn. 60, 409-417 (1991).

29. R. Hirota, "Exact envelope-soliton solutions of a nonlinear wave equation," J. Math. Phys. 14, 805-809 (1973).

30. D. Anderson and M. Lisak, "Nonlinear asymmetric self-phase modulation and self-steepening of pulses in long optical waveguides," Phys. Rev. A 27, 1393-1398 (1983).

31. S. Palacios, A. Guinea, J. M. Fernandez-Diaz, and R. D. Crespo, "Dark solitary waves in the nonlinear Schrödinger equation with third order dispersion, self-steepening, and self-frequency shift," Phys. Rev. E 60, R45-R47 (1999).

32. Z. Li, L. Li, H. Tian, and G. Zhou, "New types of solitary wave solutions for the higher order nonlinear Schrödinger equation," Phys. Rev. Lett. 84, 4096-4099 (2000).

33. T. Brugarino and M. Sciacca, "Singularity analysis and integrability for a HNLS equation governing pulse propagation in a generic fiber optics," Opt. Commun. 262, 250-256 (2006).

34. U. Bandelow and N. Akhmediev, "Persistence of rogue waves in extended nonlinear Schrödinger equations: integrable Sasa-Satsuma case," Phys. Lett. A 376, 1558-1561 (2012).

35. A. Ankiewicz, J. M. Soto-Crespo, and N. Akhmediev, "Rogue waves and rational solutions of the Hirota equation," Phys. Rev. E 81, 046602 (2010).

36. N. Akhmediev, V. I. Korneev, and N. V. Mitskevich, "Modulation instability of CW signal in an optical fiber with the 3rd-order dispersion,” Izv. Vyssh. Uchebn. Zaved. Radiofiz. 33, 95-100 (1990); Radiofiz 33, 111-117 (1990). 